\title{
Multiple Model Predictive Control on a drainage canal system
}

\author{
Peter-Jules van Overloop*, Steven Weijs, Sjoerd Dijkstra \\ Faculty of Civil engineering and Geoscience, Delft University of Technology, Stevinweg 1, 2628CN Delft, The Netherlands
}

Received 15 February 2006; accepted 11 June 2007

Available online 27 August 2007

\begin{abstract}
Model Predictive Control has been implemented on a large drainage canal system in the Netherlands. This water system can be represented as a reservoir with uncertain inflow due to rainfall runoff and a water level that has to be kept within a certain range by a control flow that is limited in capacity. Tests demonstrate that Model Predictive Control outperforms feedback and feedforward controls. To deal with uncertainty in the expected inflow, Multiple Model Predictive Control (MMPC) is proposed. This controller minimizes an objective function in which the risk of damage is used by applying different scenarios to multiple identical models.

(C) 2007 Elsevier Ltd. All rights reserved.
\end{abstract}

Keywords: Model predictive control; Multiple model optimisation; Multi-objective optimisations; Uncertainty; Open water systems; Risk

\section{Introduction}

In areas with considerable amounts of precipitation, water systems are designed to drain water out of the area into an adjacent river or sea. The precipitation falls on the land, resulting in a run-off into the system through infiltration via ground water and more direct from precipitation on the water surface and surface run-off. The water system consists of storage and conveyance canals and structures to manipulate the flows in these canals. The sluice gates and pumps that discharge water out of the system are the most important structures, as their capacity defines the maximum drainage capacity of the system. The capacity of the structures is designed to be sufficient to deal with regular storm events. Heavy storm events cause inflows that exceed drainage capacity, resulting in rising water levels. This rise could eventually lead to overtopping of dikes and embankments, causing inundation with damage to farm land and communities. Consequently, the storage capacity within the water system needs to be utilized as much as possible for these heavy storm events. By lowering the water level before a storm event actually starts, extra storage is created that can be utilized to store the run-off water to avoid high water levels. On the

\footnotetext{
*Corresponding author. Tel.: + 31620368817 ; fax: + 31152785559 .

E-mail address: p.j.vanoverloop@tudelft.nl (P.-J. van Overloop).
}

other hand, water levels cannot be lowered too much due to the risk of collision of dikes. Also, changes to the structure's settings need to be minimized to avoid wear and tear to the structures and strong transition waves in the canals. The management of the water levels between these minimum- and maximum-allowed water levels is normally performed by an operator, who needs to have extensive experience with the system. He judges the present state of the water system and the precipitation forecast for the coming period to decide on the control actions. In his decision, he uses an estimate of the effect that the storm event has on the water levels and the limited capacity of the structures.

As requirements from the society in which the drainage system functions, increase over time and the storm events tend to become more intense, the operator needs to be supported in his difficult task by the implementation of a control system. The objective of the control system is to keep the water levels in the canal system close to target level, given the limited capacity of the structures.

Over the last few decades, the type of controllers applied on water systems have evolved from feedforward, feedback, feedback in combination with feedforward towards more advanced control methods such as Model Predictive Control (Camacho \& Bordons, 1999; van Overloop, Schuurmans, \& Brouwer, 2003; Qin \& Badgwell, 2003). (Malaterre, Rogers, \& Schuurmans, 1998) gives a thorough 
classification of all types of control methods used on water systems. This trend of implementing more advanced control systems is in line with the increasing capability over the last decade to implement controllers in 'real time'. To a large extent, this is the result of the increased computational power of computers and microprocessors. Nowadays, by making use of predictions, Model Predictive Control makes it possible to anticipate on expected future problems resulting from the limited capacity of the structures, by using an internal process model to calculate the future state of the system. In the Model Predictive Controller, a trade-off is made between water level deviations from setpoint and the required changes in structure setting. This way of controlling the drainage system resembles the way operators manage the system. In 2003, a Model Predictive Controller has been implemented in the Decision Support System of the drainage canal system of Waterboard Delfland in the Western part of the Netherlands (Schuurmans, van Overloop, \& Beukema, 2003). Every $15 \mathrm{~min}$, this automated system calculates the optimal control actions for the structures for the next $24 \mathrm{~h}$ and communicates these actions to the operator through a user interface. When the operator accepts the advice, it is sent to the structures that effectuate the control actions automatically.

The use of predictions implies the introduction of uncertainties. The inflow in the canal system, that cannot be measured directly, is influenced by uncertainty in (forecasted) precipitation and in parameters of the hydrological run-off process. A way to gain insight in the extent of the uncertainties is to use an ensemble prediction system. Here, the predictions are computed in a number of runs. For each run, the parameters of the model are slightly changed. The result is a range of scenarios providing an estimate of the outer bounds and the probabilities of the predicted inflow.

A fundamental question is how to use the uncertainties represented by ensemble predictions in the computation of the control actions. A method that is sometimes used to calculate the control actions under uncertainty is chanceconstrained programming (Charnes \& Cooper, 1959). Due to heavy non-linear processes, especially in the rainfallrunoff part, this method is hard to apply to the Delfland case. Furthermore, this method will result in control actions that try to come as close as possible to the chance constraint, while in the Model Predictive Controller as applied in this article, the water levels should be kept to setpoint as close as possible. This requires usage of the deviation of water levels from setpoint or, preferably, the derived variable damage in the objective function of the Model Predictive Controller. Another way to incorporate the uncertainty in the optimization problem is to minimize the risk resulting from the uncertain inflows. Previous work considers risk in optimization and control problems (Zafra-Cabeza, Ridao, \& Camacho, 2004, 2005; Zafra-Cabeza, Ridao, Camacho, Kempf, \& Rivera, 2007). In the case presented in this article, however, the risk itself is the direct result of an uncertain disturbance that varies in time. The control actions influence both the probability and the damage associated with extreme water levels. The product of these two variables is part of the objective function. When the total risk of all scenarios is minimized, the optimal control actions are computed, taking into account the uncertainties in the system. In this article, a Multiple Model approach (Murray-Smith \& Johansen, 1997) is proposed to incorporate the risk of damage in computing the control actions using a probabilistic approach.

\section{Model of drainage canal system}

A simple model of a drainage canal system in a low-land area is used to illustrate the functioning of control on water systems. In Fig. 1 the water system is shown, in which $h$ is the average water level ( $m$ with respect to mean sea level), $Q_{c}$ is the control flow that pumps water out of the system $\left(\mathrm{m}^{3} / \mathrm{s}\right)$ and $Q_{d}$ is the disturbance rainfall-runoff inflow $\left(\mathrm{m}^{3} / \mathrm{s}\right)$. This disturbance flow originates from the precipitation that finds its way through the ground water and overland flow to the water system and the direct precipitation on the water surface.

The discrete time invariant process model used is given by

$h(k+1)=h(k)-\frac{T_{c}}{A_{s}} Q_{c}\left(k-k_{d}\right)+\frac{T_{c}}{A_{s}} Q_{d}(k)$,

where $A_{s}$ is the average storage area $\left(\mathrm{m}^{2}\right), T_{c}$ is the control time step (s), $k_{d}$ is the number of delay steps between control action and change in average water level and $k$ is the time step index. The canal system can be modelled as a large reservoir because the storage canals are wide and well interconnected. This type of water system model is referred to as the Integrator Delay model (Schuurmans, 1997). Most of the drainage systems in low-land areas can be modelled in this way, as they are characterized by wide canals with a flat bottom slope. To demonstrate the validity of the simplified model, measurements of the Delfland water system are compared to simulation results of the

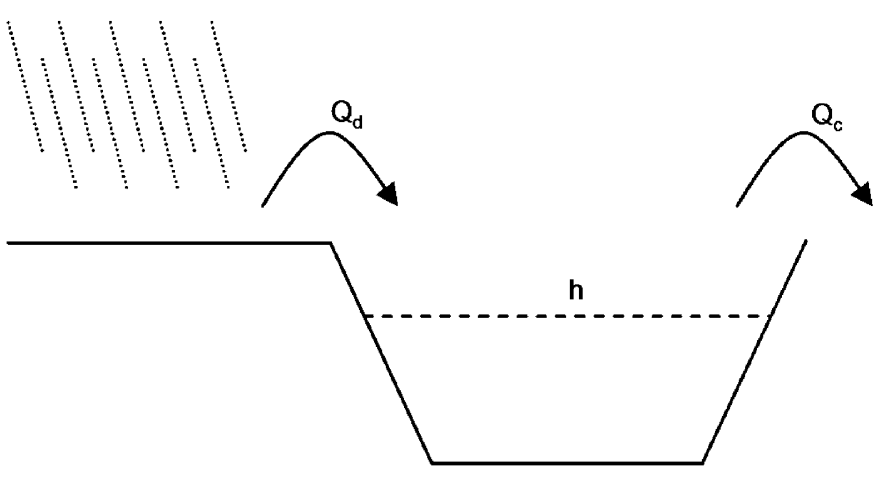

Fig. 1. Schematization of drainage water system. 
simplified model. This water system is controlled by the Model Predictive Controller as described in this article. Table 1 presents the parameters of the simplified model as derived from the actual water system. Figs. 2 and 3 present the comparison of the actual water system and the model over 12 days starting from 7 January 2006. Note that the reference level in this period is set to $-0.475 \mathrm{~m}$ with respect to mean sea level. In Fig. 2, the precipitation over this period is shown together with the resulting rainfall runoff. This flow is the disturbance to the reservoir model and is calculated based on a non-linear deterministic

Table 1

Model and controller parameters

\begin{tabular}{lll}
\hline Parameter & Symbol & Value \\
\hline Storage area & $A_{s}$ & $73,00,000 \mathrm{~m}^{2}$ \\
Control time step & $T_{c}$ & $900 \mathrm{~s}$ \\
Delay step & $k_{d}$ & 1 \\
Water level setpoint & $R$ & $-0.40 \mathrm{mMSL}$ \\
Minimum water level & $h_{\min }$ & $-0.55 \mathrm{mMSL}$ \\
Maximum water level & $h_{\max }$ & $-0.30 \mathrm{mMSL}$ \\
Minimum control flow & $Q_{c, \min }$ & $0 \mathrm{~m}^{3} / \mathrm{s}$ \\
Maximum control flow & $Q_{c, \text { max }}$ & $75 \mathrm{~m}^{3} / \mathrm{s}$ \\
Prediction horizon & $N$ & $97(24 \mathrm{~h})$ \\
Proportional gain & $K_{p}$ & 1106.1 \\
Integral gain & $K_{i}$ & 75.4 \\
Penalty weight on $e$ & $Q_{e}$ & 25 \\
Penalty weight on $\Delta e$ & $Q_{\Delta e}$ & 400 \\
Penalty weight on $e_{\text {zone }}$ & $Q_{e_{\text {zone }}}$ & $2,50,000$ \\
Penalty weight on $\Delta Q_{c}$ & $R_{\Delta Q_{c}}$ & 0.01 \\
Penalty weight on $u_{\text {zone }}$ & $R_{\text {uzone }}$ & $1 \mathrm{e}-12$ \\
\hline
\end{tabular}

rainfall-runoff model. Especially in this sub-system, uncertainties play an important role. Both the prediction of the precipitation and parameters such as the initial groundwater level, infiltration capacity and saturation of the soil, are uncertain to a considerable extent. The lower part of Fig. 2 represents the actual (solid line) and the modelled (dotted) control flow that is sent to the structures in order to maintain the water levels close to reference level. The upper part of Fig. 3 shows the water levels as measured at various locations in the Delfland water system. From these measurements, the weighted average is calculated as shown in the lower part of Fig. 3 (solid line). Here, also the modelled water level is shown (dotted line) in comparison to the actual water level. It can be seen that the reservoir model is an accurate representation of the actual water system.

\section{Control on drainage canal system}

The control flow $Q_{c}$ is used to keep the water level in the reservoir model between the minimum- and maximumallowed water levels. Different control methods can be applied to manipulate this control flow. To show the reason why more advanced control methods are required to control large drainage systems, three general controllers are tested on their capability to utilize the available storage in the canal system as much as possible. The tests are open loop tests over the prediction horizon, which means that the results are the predictions of control actions to be taken and the resulting predicted water levels. When the internal model and predictions are assumed perfect, the closed loop
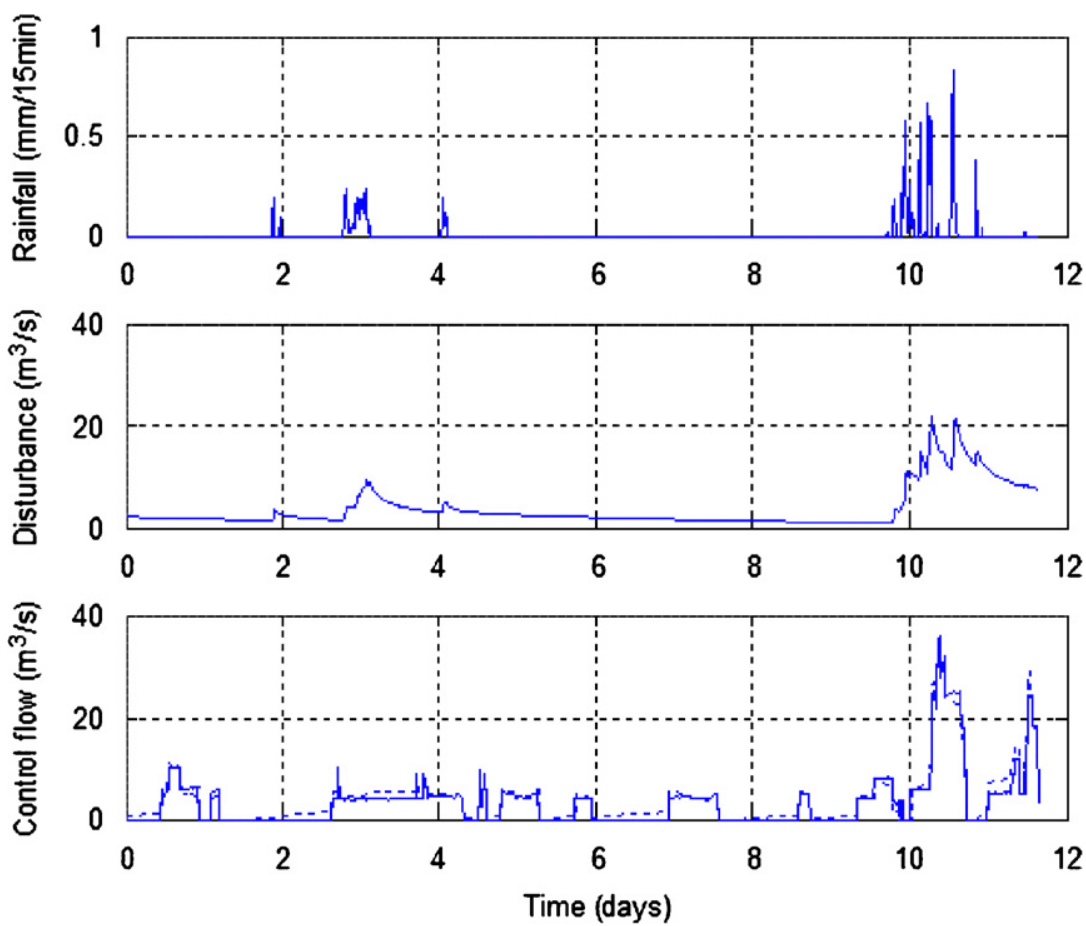

Fig. 2. Flows of actual and modelled water system. 

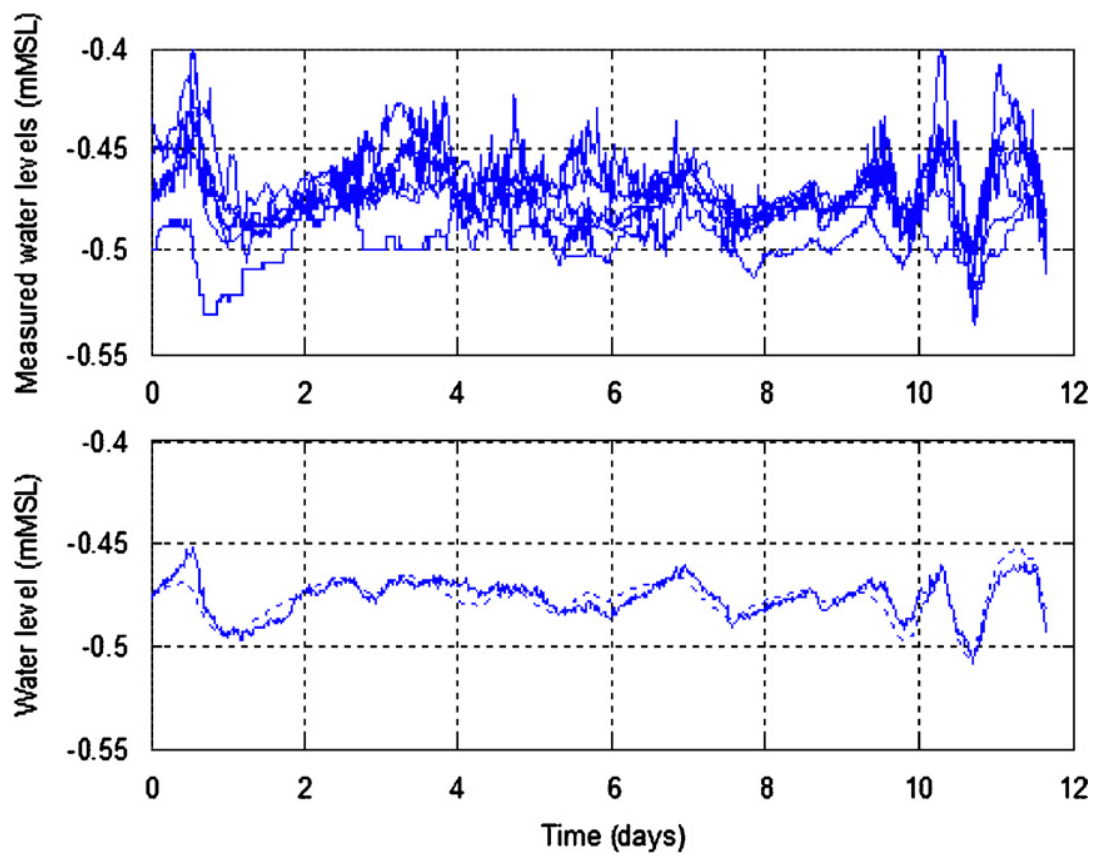

Fig. 3. Water levels of actual and modelled water system.

will give the same results. As this article only aims to show the potential of the different control methods for utilizing storage in the water system, given the predictions and the state of the water system, no closed loop tests are executed. The following controllers are tested in Matlab (Mathworks, 1992) on the Integrator Delay model of the drainage water system:

Proportional integral feedback control (Vandevegte, 1990). The water level $h$ is measured, compared to the setpoint $h_{r e f}$ and the error $e$ between these values is fed back to the control flow using a PI controller tuned with rules from (Schuurmans, 1997)

$e(k)=h(k)-h_{r e f}$,

$\Delta Q_{c}(k)=K_{p} \Delta e(k)+K_{i} e(k)$.

The computed control flow $Q_{c}$ is limited when the maximum capacity is exceeded.

Proportional integral feedback control with feedforward control (Vandevegte, 1990). The same feedback law is applied as used in the first controller and in addition to this, the most likely prediction of the disturbance run-off inflow $Q_{d \text { avg }}$ is used as feedforward signal

$\Delta Q_{c}(k)=K_{p} \Delta e(k)+K_{i} e(k)+\Delta Q_{d, a v g}\left(k+k_{d}\right)$.

The computed control flow is limited when the maximum capacity is exceeded.

Model Predictive Control (Camacho \& Bordons, 1999). The MPC uses a linear state space model with hard constraints on the input $\Delta Q_{c}$ and soft constraints on the exceedence of the water level limitations (van Overloop, 2006). When formula (1) is substracted by the same formula but now at time step $k$ and $h_{\text {ref }}$ from formula (2) is considered as constant, the change in error can be derived

$$
\begin{aligned}
h(k+1) & -h(k)=h(k)-h(k-1)-\frac{T_{c}}{A_{s}} Q_{c}\left(k-k_{d}\right) \\
& +\frac{T_{c}}{A_{s}} Q_{c}\left(k-k_{d}-1\right)+\frac{T_{c}}{A_{s}} Q_{d}(k)-\frac{T_{c}}{A_{s}} Q_{d}(k-1) \\
= & \Delta e(k+1)=\Delta e(k)-\Delta \frac{T_{c}}{A_{s}} Q_{c}\left(k-k_{d}\right)+\Delta \frac{T_{c}}{A_{s}} Q_{d}(k) .
\end{aligned}
$$

As $k_{d}$ is 1 time step (see Table 1), the state space model can be written as

$$
\begin{aligned}
& {\left[\begin{array}{c}
e(k+1) \\
\Delta e(k+1) \\
e_{\text {zone }}(k) \\
\Delta Q_{c}(k)
\end{array}\right]=\left[\begin{array}{cccr}
1 & 1 & 0 & -\frac{T_{c}}{A_{s}} \\
0 & 1 & 0 & -\frac{T_{c}}{A_{s}} \\
1 & 1 & 0 & -\frac{T_{c}}{A_{s}} \\
0 & 0 & 0 & 0
\end{array}\right] \cdot\left[\begin{array}{c}
e(k) \\
\Delta e(k) \\
e_{z o n e}(k-1) \\
\Delta Q_{c}(k-1)
\end{array}\right]} \\
& +\left[\begin{array}{cc}
0 & 0 \\
0 & 0 \\
0 & -1 \\
1 & 0
\end{array}\right] \cdot\left[\begin{array}{l}
\Delta Q_{c}(k) \\
u_{\text {zone }}(k)
\end{array}\right]+\left[\begin{array}{c}
\frac{T_{c}}{A_{s}} \\
\frac{T_{c}}{A_{s}} \\
\frac{T_{c}}{A_{s}} \\
0
\end{array}\right] \cdot\left[\Delta Q_{d}(k)\right] \\
& Q_{c}(k) \geqslant Q_{c, \min }(k), \\
& Q_{c}(k) \leqslant Q_{c, \max }(k), \\
& u_{\text {zone }}(k) \geqslant h_{\min }(k)-h_{\text {ref }}, \\
& u_{\text {zone }}(k) \leqslant h_{\max }(k)-h_{\text {ref }},
\end{aligned}
$$


where $k$ is the time step index, $e$ is the deviation between water level $h$ and the fixed setpoint $h_{r e f}(\mathrm{~m}), \Delta e$ is the change in water level deviation (m), $e_{z o n e}$ is the water level outside of the allowed margin (zone) around setpoint $(\mathrm{m}$ ) given by the minimum and maximum allowed water levels and $u_{z o n e}$ is the signal that is subtracted from the water level deviation to make $e_{z o n e}$ either zero or a value corresponding with the exceedence of the margin around setpoint (van Overloop, 2006). This signal $u_{\text {zone }}$ has no physical meaning. It is equal to $e$, when $e$ is within the minimum- and maximum-allowed levels around setpoint and limited to these minimum and maximum deviations, when $e$ is outside the allowed margin (violation of soft constraints). Fig. 4 gives an example of the variables $e, u_{z o n e}$ and $e_{z o n e}$ for a violation of a water level constraint.

The objective function $J$ that is minimized is

$$
\underset{\min \Delta Q_{c}}{J}=\sum_{i=0}^{n}\left\{\begin{array}{c}
e_{i}^{\mathrm{T}} \cdot Q_{e} \cdot e_{i}+\Delta e_{i}^{\mathrm{T}} \cdot Q_{\Delta e} \cdot \Delta e_{i} \\
+e_{\text {zone }, i}^{\mathrm{T}} \cdot Q_{e_{\text {zone }}} \cdot e_{\text {zone }, i} \\
\quad+u_{z o n e, i}^{\mathrm{T}} \cdot R_{u_{z \text { zone }}} \cdot u_{z o n e, i}+\Delta Q_{c, i}^{\mathrm{T}} \cdot R_{\Delta Q_{c}} \cdot \Delta Q_{c, i}
\end{array},\right.
$$

where $n$ is the length of the prediction horizon, $Q_{e}$ is the penalty weight on the water level deviation, $Q_{\Delta e}$ is the penalty weight on the change of water level deviation, $Q_{e_{z o n e}}$ is the (high) penalty weight on the water level deviation outside of the margin around setpoint, $R_{u_{z o n e}}$ is the (very low) penalty weight on the virtual signal that is subtracted from the water level deviation to make $e_{z o n e}$ either zero or a value that corresponds with the exceedence of the margin around setpoint. The objective function is a summation of penalties. In this article, the summed penalties on the water level deviation related variables in the objective function are considered to be the definition of damage. This may be assumed because the controller resembles the way operators manage the water system and they implicitly try to minimize costs due to inundation, failure of dikes and wear of structures. The optimal predicted control flows, that give a minimum objective function value, are computed using a quadratic programming interior point algorithm (Wright, 1997).

The parameters used in the model and controllers are given in Table 1. These parameters are derived from the actual water system of Water Board Delfand in the
Netherlands. The canal system of Delfland is representative for most of the large drainage systems in low-land areas. Although the size of different drainage canal systems may vary, the basic dynamics are the same as in the presented example. The values for the penalty weights are derived by normalizing all terms in the objective function to 1 when maximum-allowed values for the variables are assumed. These values are $0.2 \mathrm{~m}, 0.05 \mathrm{~m} /$ time step, $0.002 \mathrm{~m}$, $\left(10 \mathrm{~m}^{3} / \mathrm{s}\right) /$ time step and $10,00,000 \mathrm{~m}$ for the variables $e, \Delta e$, $e_{z o n e}, \Delta Q_{c}$ and $u_{z o n e}$, respectively.

The results of the three control methods are presented in Figs. 5-7. The forecasted precipitation is the actual precipitation of September 131998 6:00 that fell in the Western part of the Netherlands. This event was one of the heaviest storms in the past 100 years in this area.

In Fig. 5, the result of the proportional integral feedback controller is shown. The first graph is the disturbance flow $Q_{d}$. The second graph is the computed control flow $Q_{c}$, while the third graph is the resulting water level $h$. The controller starts to react on the disturbance after this has caused the water level to rise. In fact, the control action is too late. When the disturbance inflow causes the water level to rise, the control flow increases in order to bring the water level back to setpoint. As the capacity of the control flow is limited to $75 \mathrm{~m}^{3} / \mathrm{s}$, the water level rises higher than the maximum allowed water level.

Fig. 6 is the result of the same proportional integral feedback controller in combination with a feedforward controller. The feedforward controller computes the effect that the disturbance has on the water level and compensates for this by counter acting on this effect. It starts to discharge one time step before the disturbance takes place, as the delay time from control action to average water level change is one time step. Apart from this time shift, the course of the control flow is similar to the disturbance flow, as this controller tries to keep the resulting water level deviation exactly to zero. Only when the disturbance flow becomes higher than the maximum control flow, the water level rises and violates the maximum-allowed water level. This controller reacts faster, but still does not satisfy the requirement on the controlled water levels.

Fig. 7 shows that the Model Predictive Controller results in a solution that does not violate the constraints. As the

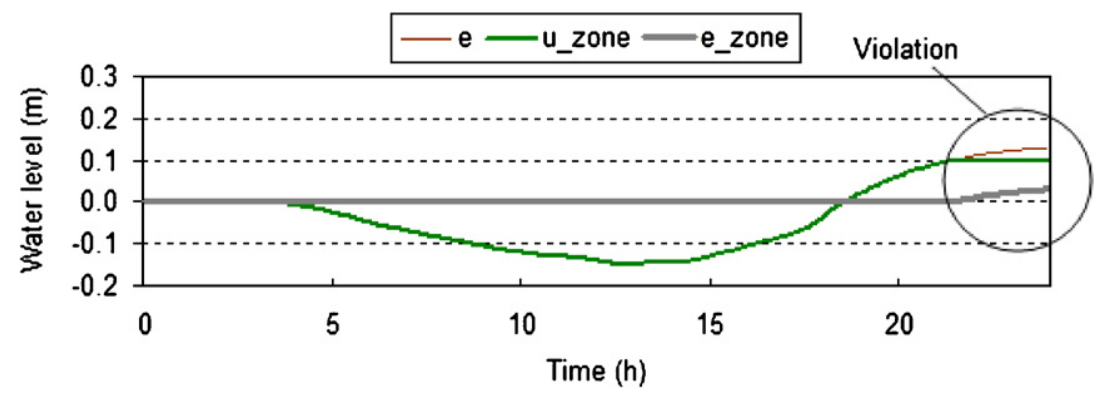

Fig. 4. Virtual variables required to construct soft constraint. 

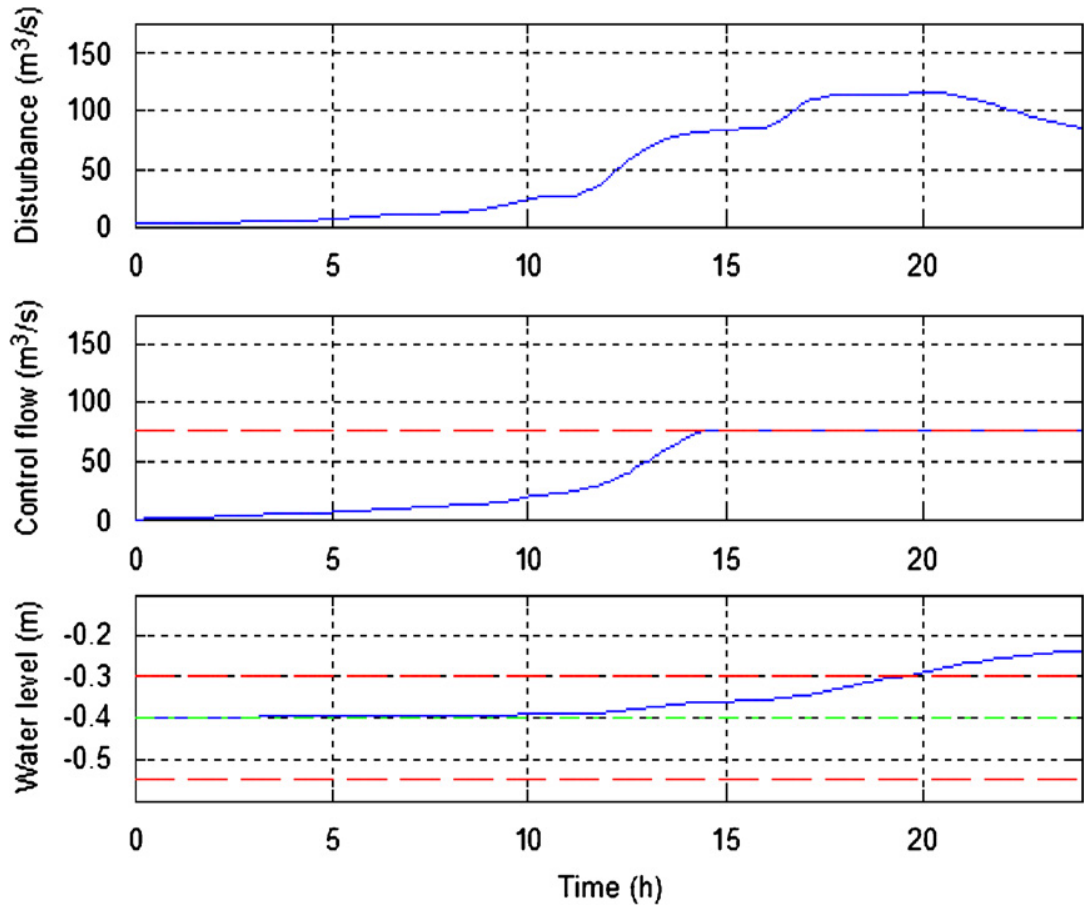

Fig. 5. Feedback control.
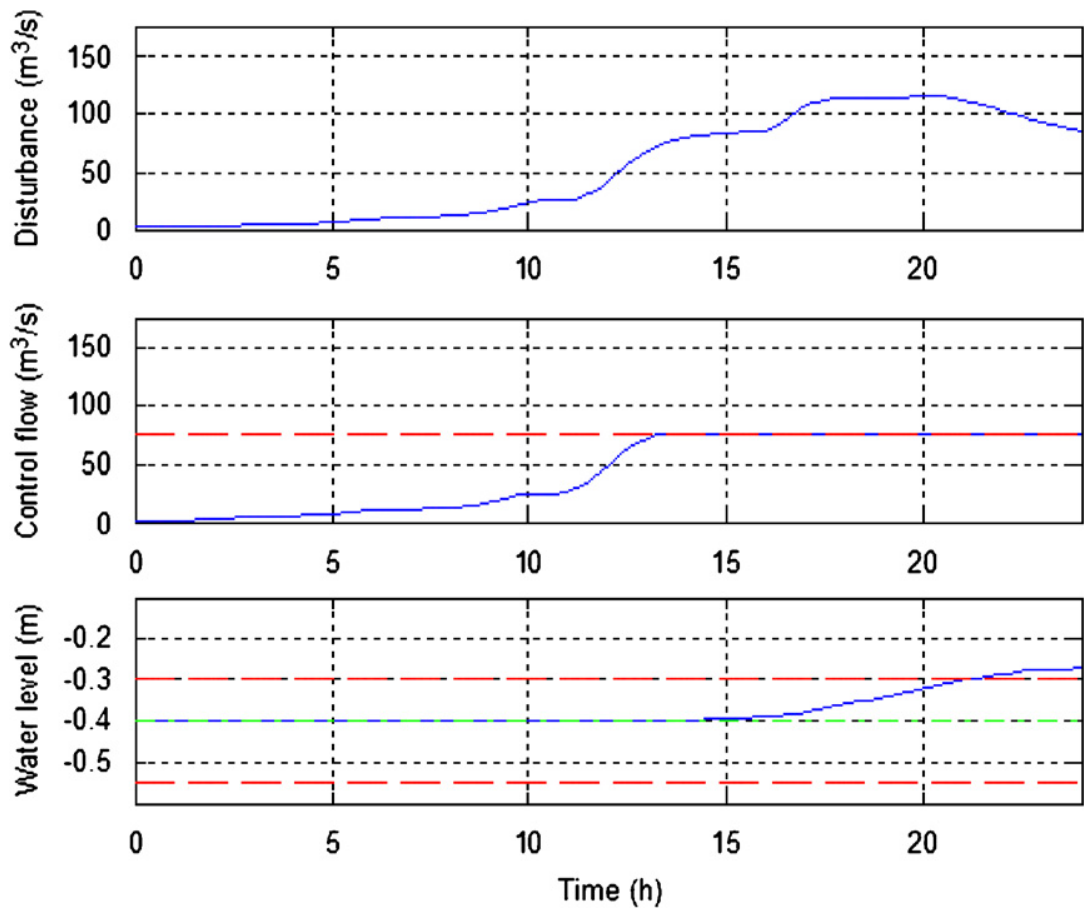

Fig. 6. Feedback and feedforward control.

capacity of the pump is limited, the controller does not function as a straightforward feedforward controller. Instead, the control flow is augmented before the disturbance actually takes place. By lowering the water level beforehand, extra storage is created to accommodate for the water level rise due to the inflowing disturbance.

\section{Uncertainties in controlled drainage canal systems}

Since the forecasted precipitation is uncertain and the disturbance model contains model errors as well, the disturbance inflow over the prediction horizon is uncertain. As the hydrological process is highly non-linear, white 
noise-based methods such as LQG are not an option to estimate the probability distribution of a solution. As the hydrological model is based on physical parameters, a Monte-Carlo analysis can be carried out in which these parameters are varied according to their estimated uncertainties. This analysis results in ensemble prediction scenarios. Other possibilities include the use of uncertain- ties in rainfall forecast. For the example shown in Fig. 8, 50 runs are carried out in which the parameters of the hydrological model are picked at random from normal probability distributions with mean values and standard deviations taken from expert judgement. The 50 runs are shown in the left part of Fig. 8. In the right part of Fig. 8, the 50 runs are sorted with respect to cumulative volume
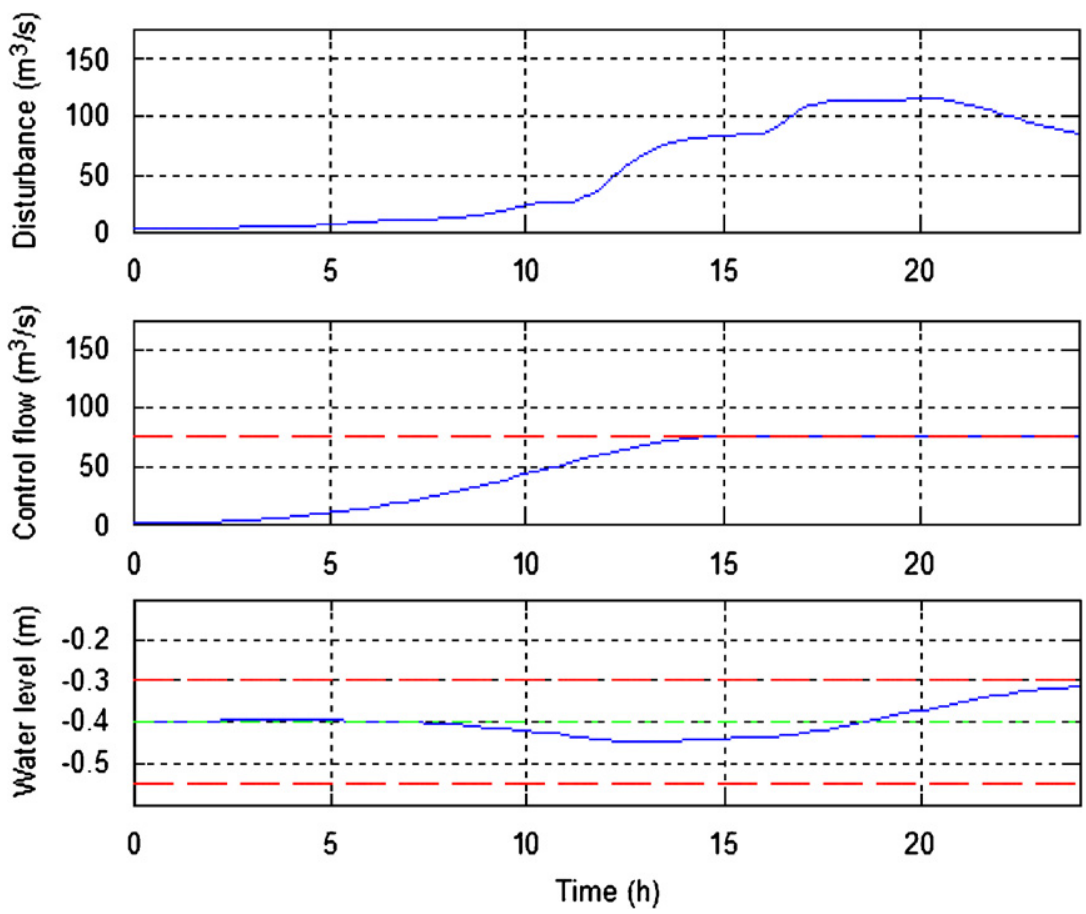

Fig. 7. Optimization by using Model Predictive Control.
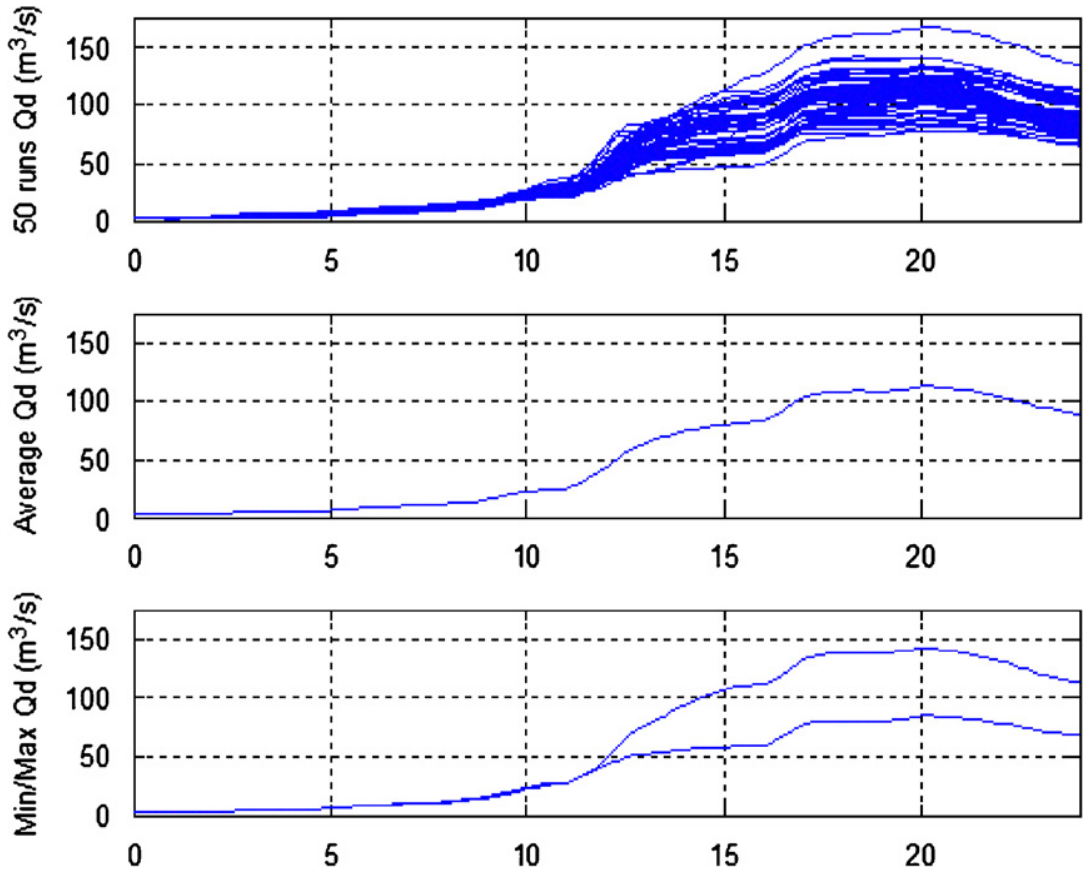

Fig. 8. Disturbance flow 50 runs and derived average and minimum. 
over the prediction horizon. The lowest 5 , middle 40 and highest 5 runs are averaged in a minimum, average and maximum scenario. Consequently, the corresponding probabilities of these scenarios are taken to be $10 \%, 80 \%$ and $10 \%$, respectively.

\section{Multiple Model Predictive Control (MMPC) on drainage canal systems}

In case the three disturbance scenarios are applied to the canal system with the same set of control actions for all scenarios, this will result in three water level trajectories. One is the maximum scenario with a small probability of occurrence, but with high damage. The second is the average scenario that is most likely to occur. The third is the minimum scenario that also has a small probability and high damage.

If one of the resulting water levels is pointed out as fully unacceptable, the corresponding disturbance can be used in the Model Predictive Controller. The control actions will then be fully concentrated on avoiding this scenario, while the other scenarios are neglected. However, if the chance of occurrence of other scenarios is much higher, the question can be posed whether this controller is too conservative. Instead, the probability combined by the damage is a more promising option. The variables that are related to the water level deviations in the objective function are considered as damage. So, the damage is assumed to be proportional to the water level deviation from setpoint. The probability $P$ is multiplied with this damage to come to a risk criterion that can be minimized

Risk $=$ Probability $\times$ Damage .

The risk criterion can be incorporated into the Model Predictive Controller by using three identical process models in parallel, fed by three different disturbance models. In the objective function, the resulting three water level deviations are weighted with an extra weight equal to their probability of occurrence $P$. Fig. 9 and formula (9) illustrate the functioning of this MMPC. that is mainly based on the most likely scenario, but in case of a large water level deviation of one of the other scenarios, the resulting control flow anticipates more on that extreme deviation. The consistency of formula (9) can be checked by assuming identical disturbance flows for the three scenarios (for example if the standard deviations of the normal distributions of all parameters in the disturbance model are set at zero). The resulting water levels of the three scenarios will then be identical. Multiplication with each probability and summation leads to the exactly same result as given by formula (6). Note that different process models, for example with minimum, average and maximum storage area $A_{s}$, can also be incorporated. This allows for robust control over all operating conditions (van Overloop, Schuurmans, Brouwer, \& Burt, 2005).

The same model as given in formula (1) and tested on the various control methods, is now tested with MMPC using different scenarios according to Fig. 8. Fig. 10 shows the open loop prediction of disturbances, the optimal control flow and the water levels. The results of MMPC have a lower final water level of the average scenario compared to the regular Model Predictive Controller. This is the result of the maximum scenario violating the maximum allowed water level. The soft constraint on that violation makes the control flow start sooner with discharging out of the water system. This results in lower water levels for all scenarios. The fact that the maximum-allowed water level is violated does not pose a problem, as the probability that it will happen is small.

Interviews with operators reveal that the result of the MMPC resembles the way that the operators manage the water system. When the expected inflow is uncertain, for example due to contradictive weather forecasts from different meteorological institutes, they tend to keep the water levels lower. Based on the uncertain predictions, the operators consider high water levels with corresponding high damage due to extreme precipitation probable. In that manner, the operators are controlling the risk of damage in a similar way as MMPC.

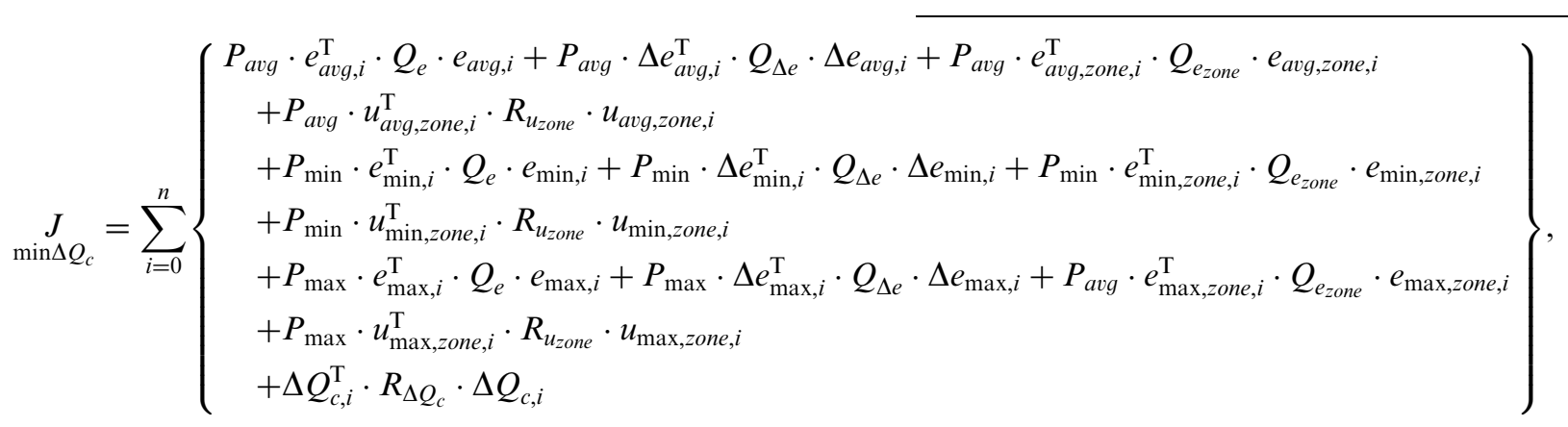

where $P_{\text {avg }}$ is the probability of the average scenario, $P_{\min }$ is the probability of the minimum scenario and $P_{\max }$ is the probability of the maximum scenario. The values for $P_{\text {avg }}$, $P_{\min }$, and $P_{\max }$ are taken at $80 \%, 10 \%$ and $10 \%$ respectively. The optimization results in a control flow
The MMPC is a simple extension of the normal Model Predictive Controller as only copies of the process model are used. The only consequence is that the matrix sizes increase with a factor of maximum $3^{2}$. This increases the computational time to solve the optimization. 

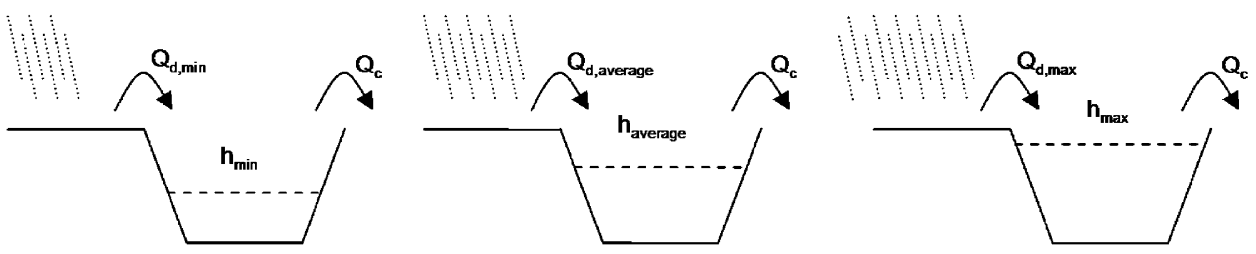

Fig. 9. Three models with different disturbance scenarios.
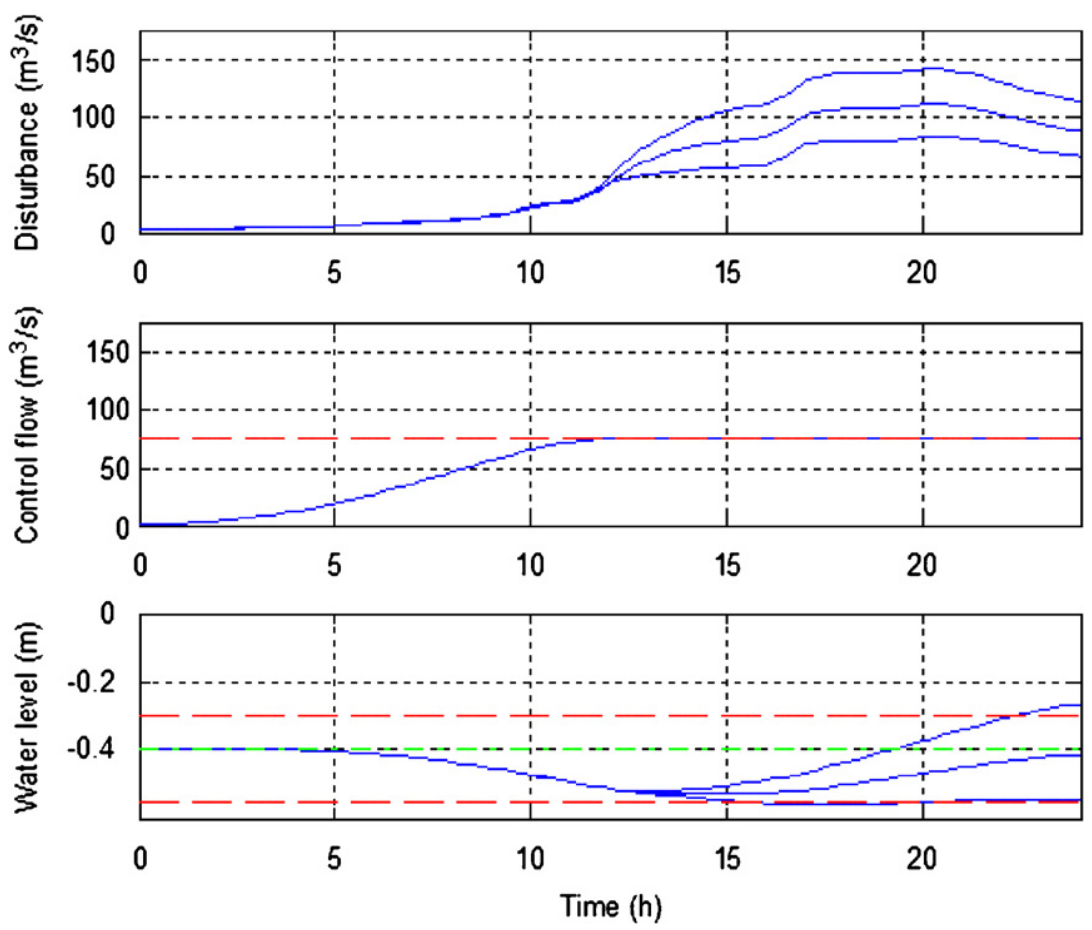

Fig. 10. Result of MMPC of one optimization step.

\section{Conclusions}

To control drainage canal systems in extreme conditions, the available storage needs to be utilized as much as possible. When heavy precipitation falls, feedback control is less capable of avoiding exceedence of the maximum water level than feedback control in combination with feedforward. Model Predictive Control outperforms feedback and feedback in combination with feedforward, as it can anticipate predicted disturbance beforehand, taking the limited capacities of the structures into account.

Since the predictions are uncertain, a methodology is applied that uses the risk approach in MPC that penalizes the variables related to the water level deviations multiplied by the probability of occurrence. A minimum, average and maximum scenario is computed in an ensemble prediction system. These scenarios are applied on three identical, parallel models on which one set of control flows is computed by a MMPC. The MMPC results in control actions taking the uncertainties into account. This has an advantage over control methods that only consider the average prediction scenario. Other scenarios can have a low probability, but may result in very high damage.
The methodology allows for structural application in a wide variety of problems that exist in the control of water systems, such as uncertain precipitation, uncertain process models and different constraints on different scenarios. Since any disturbance model and process model can be used, MMPC can be applied to other types of water systems such as control of large reservoirs, irrigation canals, etc.

\section{Acknowledgements}

The authors would like to express their gratitude to the operators of the Water Board of Delfland for providing the measurement data for this research.

\section{References}

Camacho, E. F., \& Bordons, C. (1999). Model predictive control. New York: Springer.

Charnes, A., \& Cooper, W. W. (1959). Chance-constrained programming. Management Science, 6, 73-79.

Malaterre, P. O., Rogers, D. C., \& Schuurmans, J. (1998). Classification of canal control algorithms. Journal of Irrigation and Drainage Engineering, 124, 3-10. 
Mathworks. (1992). Matlab user guide. Natick

Murray-Smith, R., \& Johansen, T. A. (1997). Multiple model approaches to modelling and control. London: Taylor \& Francis.

van Overloop, P. J. (2006). Model predictive control on open water systems. Ph.D. dissertation. Delft University of Technology, The Netherlands.

van Overloop, P. J., Schuurmans, W., \& Brouwer, R. (2003). Model predictive control of water systems in the Netherlands. In USCIDproceedings International Conference, Phoenix.

van Overloop, P. J., Schuurmans, J., Brouwer, R., \& Burt, C. M. (2005). Multiple model optimization of PI-controllers on canals. Journal of Irrigation and Drainage Engineering, 131, 190-196.

Qin, S. J., \& Badgwell, T. A. (2003). A survey of industrial model predictive control technology. Control Engineering Practice, 11(7), 733-764.

Schuurmans, J. (1997). Control of water levels in open channels. Ph.D. dissertation. Delft University of Technology, The Netherlands.
Schuurmans, W., van Overloop, P. J., \& Beukema, P. (2003). Delfland kiest voor volledige automatische bediening van kunstwerken. $\mathrm{H} 2 \mathrm{O}$ (in Dutch).

Vandevegte, J. (1990). Feedback control systems. New Jersey: Prentice hall.

Wright, S. J. (1997). Applying new optimization algorithms to model predictive control. Chemical Process Control-V, CACHE, AIChE Symposium, 93, 147-155.

Zafra-Cabeza, A., Ridao, M. A., \& Camacho, E. F. (2004). An Algorithm for optimal scheduling and risk assessment of projects. Control Engineering Practice, 12(10), 1329-1337.

Zafra-Cabeza, A., Ridao, M. A., \& Camacho, E. F. (2005). A stochastic predictive control approach to project risk management. In Preprints of the 16th IFAC World Congress. CD-ROM. Prague. Czech Republic. July 3-8, 2005.

Zafra-Cabeza, A., Ridao, M. A., Camacho, E. F., Kempf, K. G., \& Rivera, D. E. (2007). Managing risk in semiconductor manufacturing: A stochastic predictive control approach. Control Engineering Practice, 15, 969-984. 